C-A/AP/\#197 April 2005

\title{
Maps, electron-clouds in RHIC and first-order phase-transitions
}

P. Thieberger, U. Iriso and S. Peggs

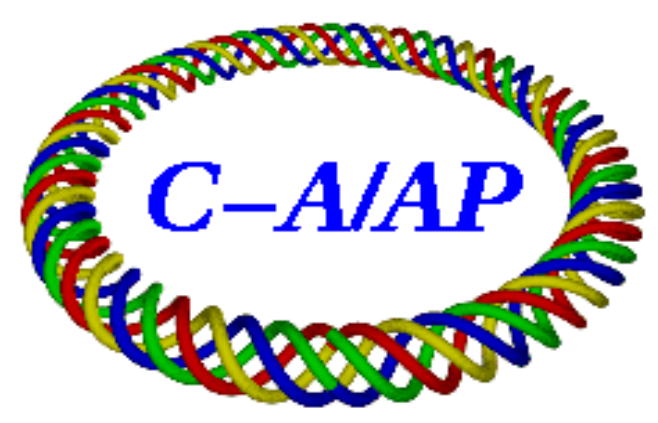

Collider-Accelerator Department Brookhaven National Laboratory Upton, NY 11973 


\title{
Maps, electron-clouds in RHIC and first-order phase-transitions
}

\author{
P. Thieberger, U. Iriso and S. Peggs
}

March 30, 2005

\begin{abstract}
A simple model is developed based on the theory of logistic maps to investigate possible explanations for sudden changes in multipacting-related outgassing rates, the so called first-order phase transitions, observed in the Relativistic Heavy Ion Collider (RHIC). Stability conditions are introduced for the map description and it is shown that a possible residual gas-related mechanism that may extend the electron survival between bunches can reproduce the type of behavior that is observed.
\end{abstract}

\section{Introduction}

The formation of electron clouds, multipacting, and the associated pressure excursions due to outgassing, limit at present the performance of machines such as the Relativistic Heavy ion Collider (RHIC) [1]. To find mitigating measures one must strive to understand the underlying mechanisms as well as possible. Achieving a detailed understanding of such complex phenomena is, however, a challenging problem to say the least, complicated by the fact that these phenomena are strongly dependent on the not always wellknown material properties such as desorption coefficient, secondary electron emission coefficients, ionization cross-sections, etc. The severe computational challenges associated with computer simulations have been met with clever approaches such as the introduction of macro-particles [2] and significant success has been achieved by the various codes [3] in understanding many aspects of this problem. But recent experimental observations at RHIC [4] and analysis for the LHC [5] indicate that additional physics might need to be taken into account. In particular, the sudden jumps in vacuum pressure observed in RHIC [6], the so-called first order phase transitions, have not been predicted by the current codes [3]. While a possible role for positive ions has been considered before [7] and found unlikely, there may be circumstances where such effects become significant.

In a novel approach [8], it was shown how results of the detailed electron cloud simulations [3] can be modeled with a few parameters by using the theory of logistic maps. This approach leads to an improved intuitive understanding of the evolution of electron clouds and, most importantly, to a seven order-ofmagnitude reduction in computation time, which is essential for analyzing and comparing a variety of bunch patterns [8]. Since first-order phase transitions were not present in the underlying models, they were of course not reproduced by that map approach. More recently, the same authors introduced a coupled-map model [9] to take into account the interplay of electron and positive ion clouds, and thus succeeded in showing how, at least in principle, first order phase transitions may come about.

There are two general mechanisms through which positive ions may modify and enhance the evolution of electron clouds. In the first one, explored in Ref. 9, the positive ions partly neutralize the electron space charge, and thus allow the bunch-to-bunch electron multiplication to continue further than in the absence of positive ions, before the gain is limited by space charge. In the second mechanism, explored here, the positive ions lengthen the time it takes for the electron cloud to decay between bunches, thus increasing the effective bunch-to-bunch gain. 
We also introduce and explore the stability conditions that play an important role in the interpretation and understanding of map-based descriptions. We will use the simplest bunch pattern, i.e. one with equally spaced bunches, and the simplest (parabolic) map to investigate how the reduction in electron-cloud decay rate due to gas ionization may explain the observation of first-order phase transitions when studying the number of electrons as function of beam intensity. But first, to set the stage and to introduce the use of stability conditions, we analyze in detail the second order phase transition predicted [8] to occur in the absence of residual gas effects.

\section{Second order phase transitions}

Following Ref. 8 , we write the electron density $\boldsymbol{\rho}_{\mathbf{m}+\mathbf{1}}$ just after bunch $\mathrm{m}$ passes as function of $\boldsymbol{\rho}_{\mathbf{m}}$, which is the density after the previous bunch passed:

$\rho_{\mathrm{m}+1}=\mathrm{a} \rho_{\mathrm{m}}+\mathrm{b} \rho_{\mathrm{m}}^{2}$

where $\mathbf{a}$ and $\mathbf{b}$ are functions of the beam parameters and also of the beam pipe characteristics. In Ref. 8 somewhat better fits are obtained with cubic maps, but at the moment this is not important since we only want to see how first-order phase transitions may come about. The same as in [8], a it is the bunch-tobunch gain for small $\boldsymbol{\rho}$ values, and $\mathbf{b}$, which is negative, may represent the space-charge effect that reduces the gain at large $\rho$ values.

A necessary condition for a steady-state solution is:

$\rho_{\mathrm{m}+1}=\rho_{\mathrm{m}}$

There are two such solutions of (1) that satisfy this necessary condition:

$\rho_{\mathrm{m}+1}=\rho_{\mathrm{m}}=0$

and

$\rho_{\mathrm{m}+1}=\rho_{\mathrm{m}}=\rho_{\mathrm{sat}}=(1-\mathrm{a}) / \mathrm{b} \quad$ for $\mathrm{a}>1$ with $\mathrm{b}<0$

But (2), while necessary, is not sufficient. The solution must also be stable. A small perturbation in the value of $\rho_{m}$ must not lead to a larger perturbation of $\rho_{m+1}$ or the values will run away. Therefore, after equilibrium has been reached, we must have

$\mathrm{d} \rho_{\mathrm{m}+1} / \mathrm{d} \rho_{\mathrm{m}} \leq 1$

for the solution to be stable. From (1) we then get the stability condition:

$\mathrm{a}+2 \mathrm{~b} \rho_{\mathrm{m}} \leq 1$

We see then that the solution (3) $(\rho=0)$ is only stable for $a \leq 1$. At $a=1$, solution (4) takes off, and it in turn is stable because substituting the value of $\rho_{\text {sat }}$ from (4) into (6) we get:

$\mathrm{a}+2 \mathrm{~b}(1-\mathrm{a}) / \mathrm{b}=2-\mathrm{a} \leq 1$ for $\mathrm{a} \geq 1$ 
So we see that at $\mathrm{a}=1$ we have a continuous transition from solution (3) to solution (4) with a sudden change of slope, i.e. a second order phase transition. This is the conclusion reached in Ref. 8. It has been developed in greater detail here to lay the foundation for analyzing examples in the next section where we explore the possible influence of the positive ions on the electron-cloud decay.

In Fig. 1 we show the dependency of steady state values of $\boldsymbol{\rho}$ on $\mathbf{a}$, and the stability condition according to the above equations. For these plots we use a constant value of $-0.6 \mathrm{~m} / \mathrm{nC}$ for $\mathrm{b}$ which is probably a good approximation according to Fig. 4 in Ref. 8, especially for the ECLOUD simulation. Since the parameter a is a monotonously increasing function of the bunch intensity, the general behavior and topography of these curves would be similar if we used bunch intensity as the independent variable.
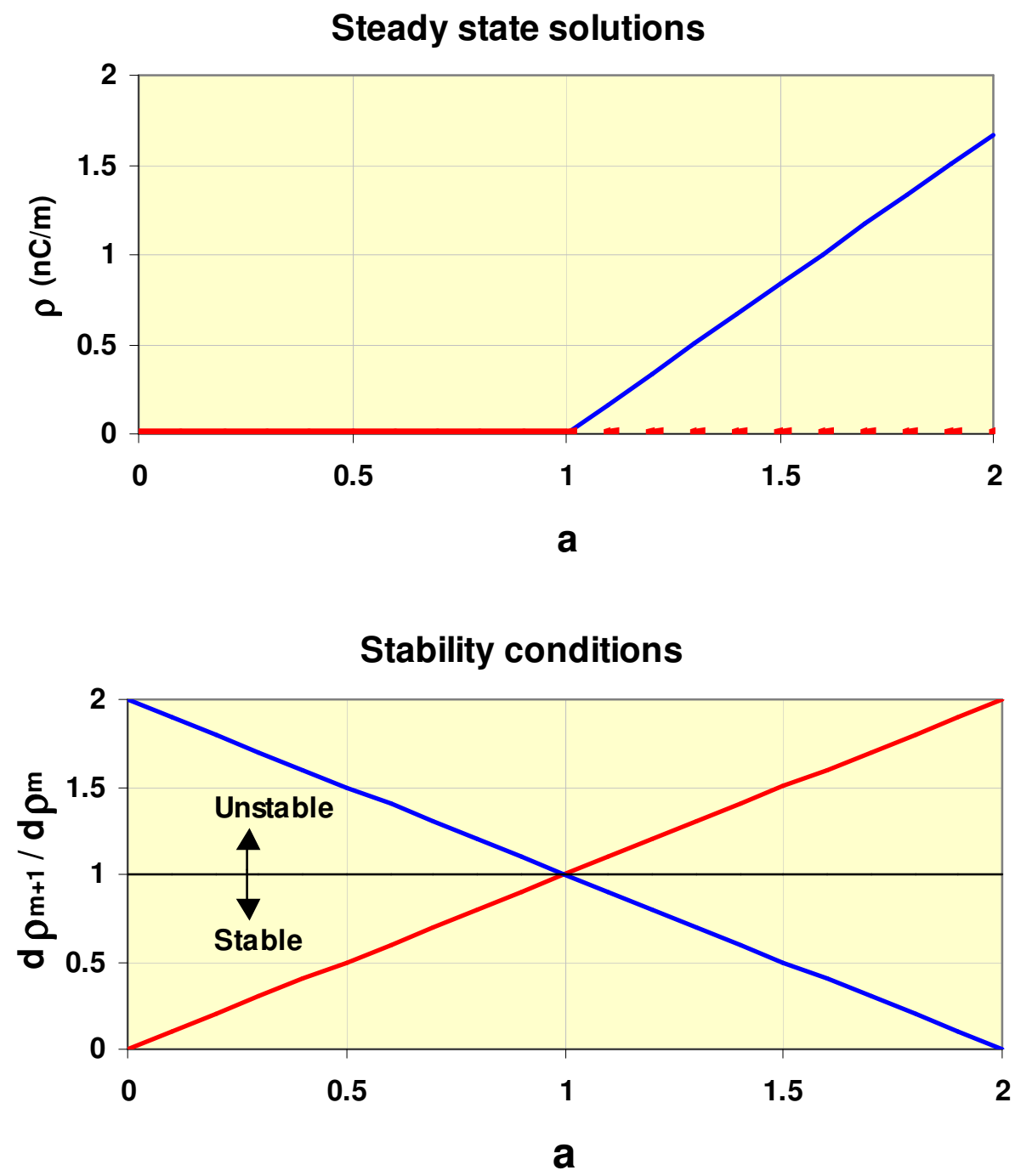

Figure 1: Steady-state solutions and corresponding stability conditions for the simple case described in the text. The $\rho=0$ solution (red lines, upper chart) is not stable for a>1 (see lower chart), and this is indicated by the dashed line in the upper chart. The non-zero solution (blue line, upper chart) is only valid for a $>1$ (positive densities) where it is also stable as can be seen from the lower chart. There is a sudden change in slope at $\mathrm{a}=1$, i.e., a second order phase transition. 


\section{A possible role of the desorbed gas}

Usually [3] a vacuum excursion that occurs in conjunction with an electron cloud is considered to be merely the consequence of electron desorption, but not an active participant in the evolution of the cloud. In Ref. 9 it was shown that this might not always be the case. Here we will explore another mechanism whereby the active participation of the desorbed gas may lead to first-order phase transitions.

First we will consider the meaning of the parameter a. For small electron densities, i.e. before space-charge effects become significant, it is the bunch-to bunch gain, which we interpret as having two factors. The first factor is the electron multiplication during the duration of the bunch, and the second factor is the electron density decay between bunches which we will assume is roughly exponential.

$\mathrm{a}=\mathrm{A} \mathrm{e}^{-\mathrm{tg} / \tau 0}$

where $\mathbf{t}_{\mathbf{g}}$ is the length of the gap between bunches, $\tau_{\mathbf{o}}$ is the electron intensity decay time in the absence of any possible density-effects we will consider below, and $\mathbf{A}$ will contain the same type of beam-intensity and pipe-material dependencies as a.

While in Ref. 9 positive ions counteract the space charge limitation of the electron gain during the presence of the bunch, here we will instead explore the possibility that positive ions may prolong the electron cloud decay time between bunches. In other words, instead of a (8), we will substitute in Eq.1 a different factor $\mathbf{a}^{\prime}$ in which the constant decay time $\tau_{\mathrm{o}}$ is replaced by a $\rho$-dependent one $\tau$.

The gas desorbed from the walls due to electron impacts will get partially ionized by the electrons, and to some extent by the ion beam, resulting in a cloud of slowly moving positive ions. The presence of such ions in the beam pipe would then help electrons survive longer, thus increasing the electron cloud decaytime. This may happen either by electron orbits being affected by the field due to the ions (partial spacecharge neutralization), or by the creation of a low-density plasma, or both. The following diagram summarizes the sequence of effects we just described:

More electrons $\rightarrow$ more gas $\rightarrow$ higher pressure $\rightarrow$ more slow ions $\rightarrow$ increased $\tau$

The simplest, first order, approximation for the $\rho$-dependence of $\tau$ is then:

$\tau=\tau_{0}+\mathrm{k} \rho$

where $\tau_{\mathbf{0}}$ is, the electron-cloud decay time for negligible electron densities and $\mathbf{k}$ is a constant that depends on such things as desorption coefficients, pumping speed, beam pipe geometry, ionization cross sections, ion velocities, etc. The real situation will be more complicated, and not only may there be higher order terms in (9) but also an explicit dependency on beam intensity, especially if it were the beam that does much of the ionizing which is probably not the case. But in the spirit of a simplified model, we will work with (9) for now.

Another factor we are ignoring is the time constant for pressure buildup, which is very long compared to the time it otherwise takes for an electron-cloud to evolve. But since we will only consider steady-state situations the final result will not be affected. In other words, we will assume that the increase in $\tau$ described by (9) takes effect immediately, while in reality it takes a while. This will have an effect on the trajectory of the system in parameter space, but not on the final states described by steady-state solutions. 
Now, instead of the parameter a (8), that doesn't depend on $\boldsymbol{\rho}$, we will have a $\boldsymbol{\rho}$-dependent coefficient $\mathbf{a}^{\prime}$ because the bunch-to-bunch gain is now being affected by the variation in decay time.

$\mathrm{a}^{\prime}=\mathrm{A} \mathrm{e}^{-\mathrm{tg} /(\tau 0+\mathrm{k} \rho \mathrm{m})}$

Using a' instead of a we thus get a new expression instead of (1):

$\rho_{\mathrm{m}+1}=\mathrm{A} \mathrm{e}^{-\mathrm{tg} /(\tau \mathrm{o}+\mathrm{k} \rho \mathrm{m})} \rho_{\mathrm{m}}+\mathrm{b} \rho_{\mathrm{m}}^{2}$

or, using (8)

$\rho_{\mathrm{m}+1}=\mathrm{a} \mathrm{e}^{[\mathrm{tg} / \tau \mathrm{\tau}-\mathrm{tg} /(\tau \mathrm{o}+\mathrm{k} \rho \mathrm{m})]} \rho_{\mathrm{m}}+\mathrm{b} \rho_{\mathrm{m}}{ }^{2}$

We will now follow the same procedure as before to find stable steady-state solutions. For this purpose, using (11), we write the new stability condition:

$\mathrm{d} \rho_{\mathrm{m}+1} / \mathrm{d} \rho_{\mathrm{m}}=\mathrm{a} \mathrm{e}^{[\mathrm{tg} / \tau \mathrm{o}-\mathrm{tg} /(\tau \mathrm{o}+\mathrm{k} \rho \mathrm{m})]}\left[1-\mathrm{k} \rho_{\mathrm{m}} \operatorname{tg}^{2} /\left(\tau_{\mathrm{o}}+\mathrm{k} \rho_{\mathrm{m}}\right)^{3}\right]+2 \mathrm{~b} \rho_{\mathrm{m}} \leq 1$

We see that, as expected, for $\mathrm{k}=0$, Eqs. 11 and 12 reduce to Eqs. 1 and 6 respectively.

To obtain, for this case, examples of electron density dependencies similar to the ones shown in Fig. 1, we use for the gap between bunches $\mathrm{tg}=103 \mathrm{~ns}$ (for a 108 bunch spacing and a $5 \mathrm{~ns}$ bunch length), and an electron decay time $\tau_{\mathrm{o}}=156 \mathrm{~ns}$ which we take as a rough approximation from Fig. 1 in Ref. 8. The assumption that the decay is exponential is in itself an approximation as mentioned above.

As before, the trivial steady state solution of (11) is zero electron density (3). The non-trivial steady-state solutions are calculated numerically using the Excel "Solver" and are shown in Figs. 2 and 3, together with the $\rho=0$ solution and the corresponding stability conditions given by (12). The parameter $\mathrm{a}=\mathrm{A} \mathrm{e}^{\text {-tg/ } / \mathrm{o}}$ has the same meaning as before, i.e. the low density overall bunch-to-bunch electron gain in the absence of gas effects, and it is a monotonically increasing function of the bunch charge.

Inspecting Fig.2, we see that for $\mathbf{k = 0}$, as expected, we get the same solutions as the ones shown in Fig.1, i.e. no residual gas effects result in a second order phase transition at $\mathbf{a}=\mathbf{1}$. For relatively small valued of $\mathbf{k}$, we still get second order phase transitions at $\mathbf{a}=\mathbf{1}$, but now reaching larger electron density values. Above a certain critical value of $\mathbf{k}$ (in this case $\mathrm{k} \cong 150 \mathrm{~ns} \mathrm{~m} / \mathrm{nC}$ ), as a exceeds the value 1 and the $\rho=0$ solution becomes unstable, there is no longer a continuous transition to the other branch (which is stable), but a discontinuous jump, i.e. a first-order phase transition. For larger values of a (corresponding to larger bunchcharge) $\boldsymbol{\rho}$ increases even more, and adopts values that are much larger than the corresponding $\mathbf{k}=\mathbf{0}$ (no gas) values. Coming down towards smaller values of $\mathbf{a}$ the system reaches values if $\mathbf{a}<\mathbf{1}$ before there is a steep drop towards $\boldsymbol{\rho}=\mathbf{0}$. i.e. the collapse of the electron cloud. This is a first order phase transition with hysteresis as can best be seen in Fig. 3 which is a magnified view of the $\mathbf{k}=\mathbf{5 0 0}$ case of Fig. 2 . 


\section{Steady state solutions}

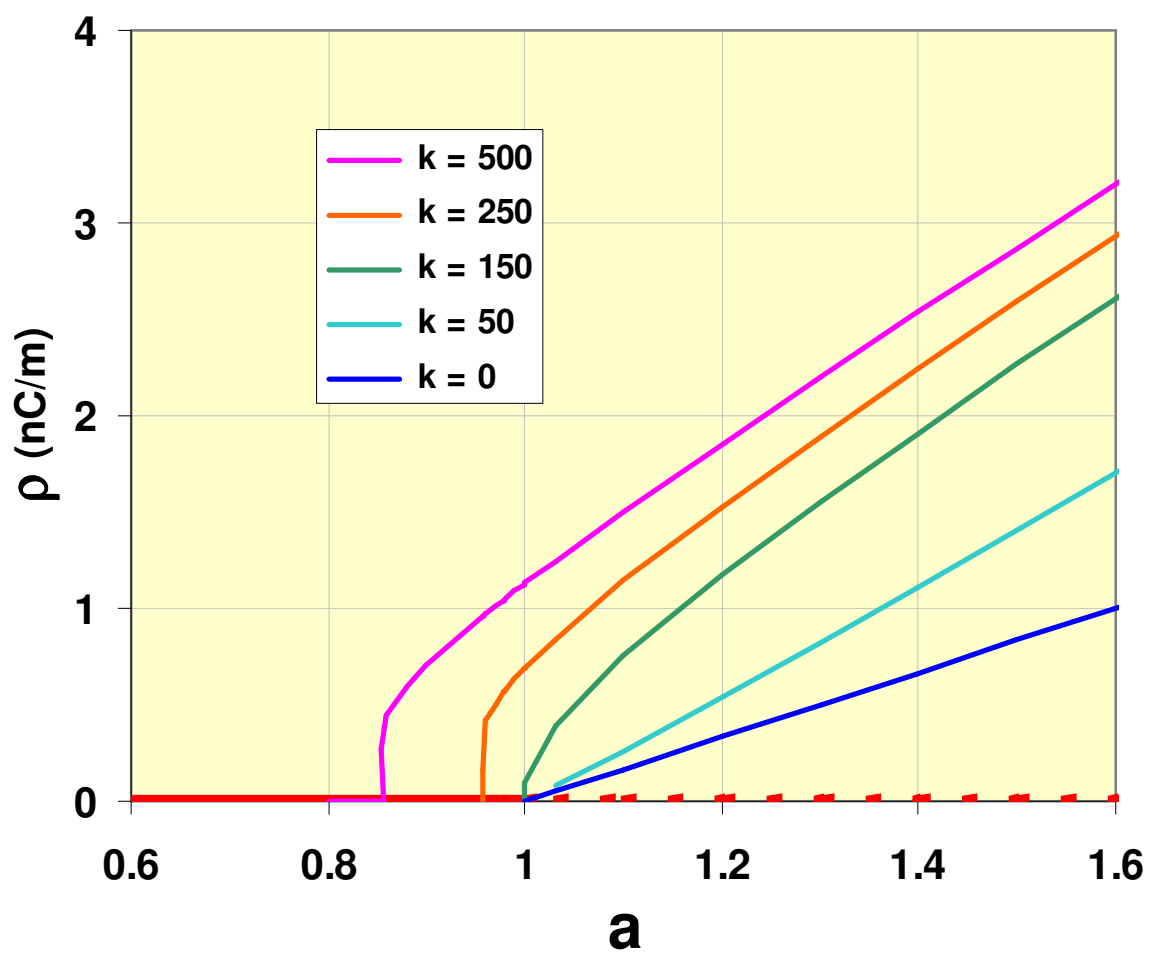

\section{Stability conditions}

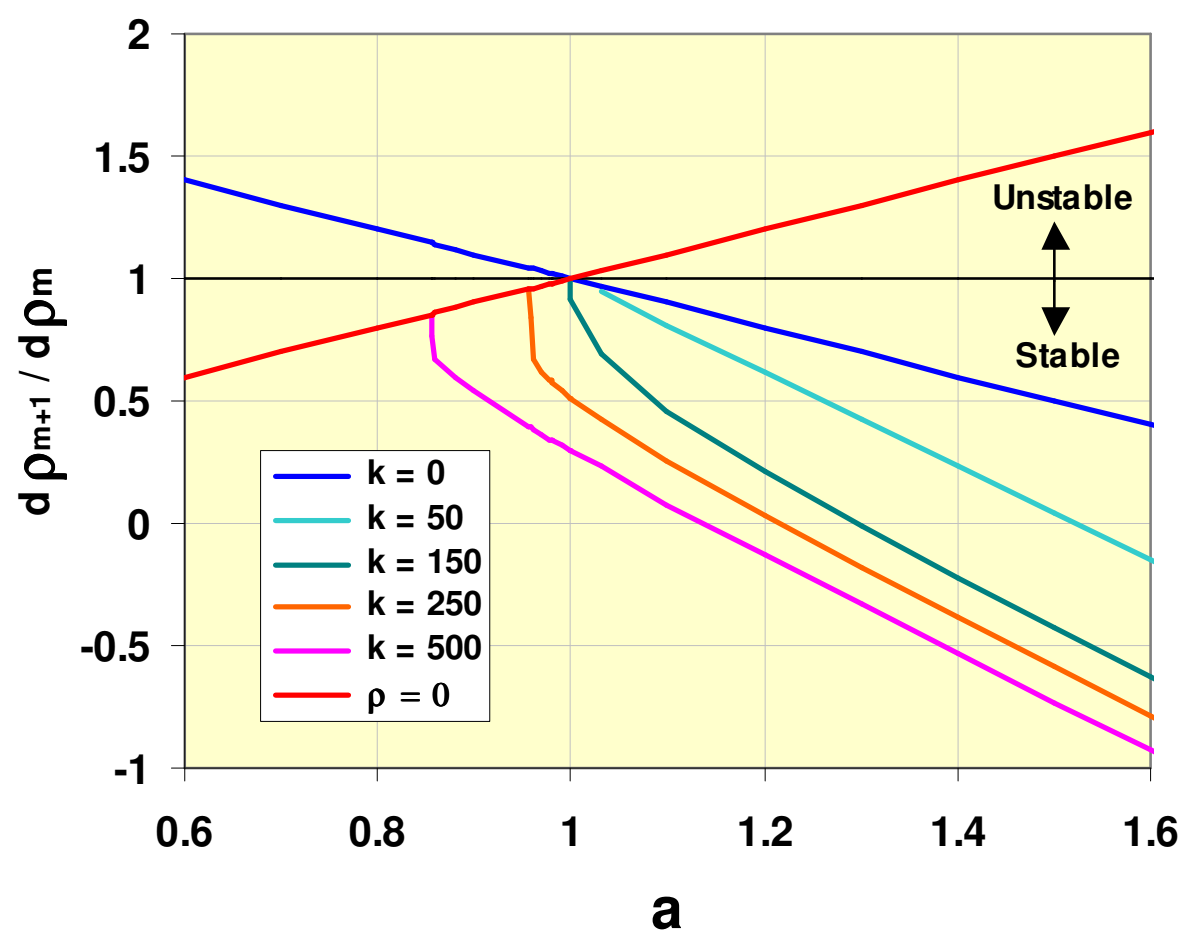

Figure 2: Steady-state solutions and stability conditions for the cases described in the text when the presence of desorbed gas slows down the electron cloud decay between bunches. The red lines for the $\rho=$ 0 solutions are the same as the ones in Fig. 1, but now apply to all the cases shown. The blue lines are for $\mathrm{k}$ $=0$ (no gas effect) and are identical to the ones shown in Fig. 1 . We see that beyond a certain value of the parameter k we get first-order phase-transitions with hysteresis (see text). 


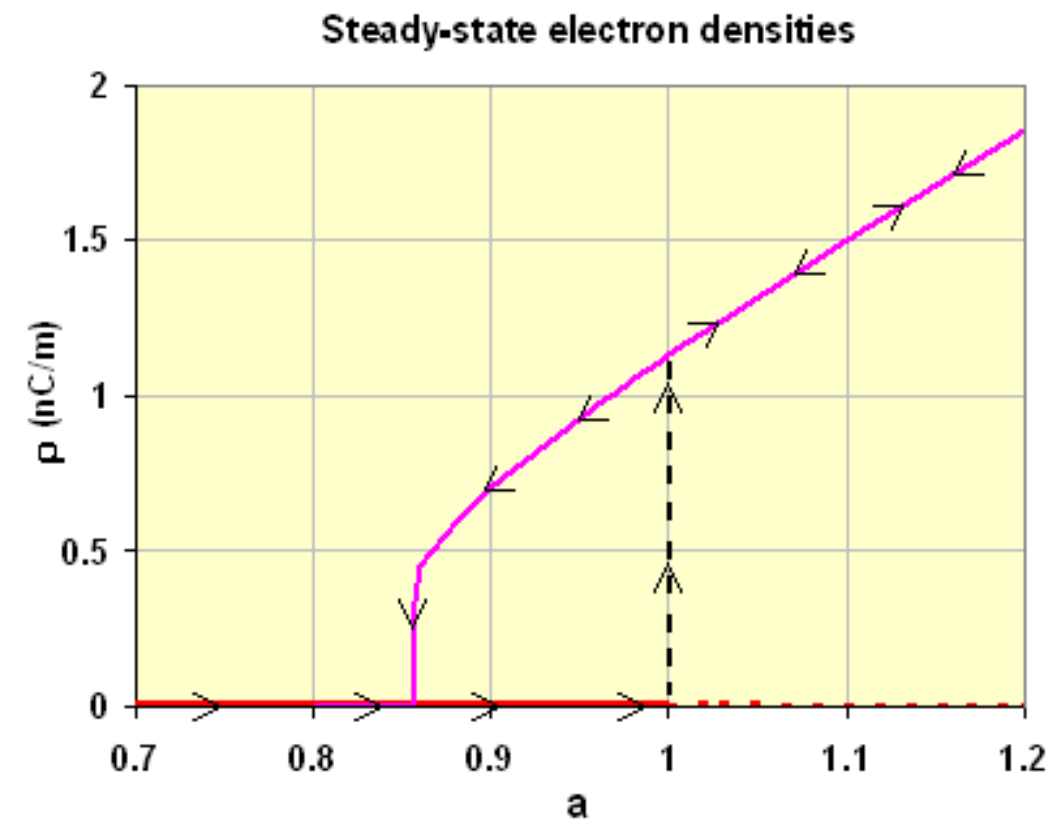

Figure 3: Close-up view of the $\mathrm{k}=500$ case in Fig. 2. The arrows indicate the evolution of the electron density as the beam intensity increases, reaches a point at $a=1$ where the $\rho=0$ solution is no longer stable, jumps to the only available stable solution (first order phase transition), and continues increasing. As the beam intensity decreases, $\rho>0$ values are maintained even below the $a=1$ point (hysteresis), until there is a steep drop to zero. It is this sharp drop that would be observed during gradual bunch decay. An upward first order phase transition can occur during filling, but is not exactly what is being modeled here (see text).

It is this collapse of the electron cloud that most likely can be correlated to such events in RHIC [6] that occur when the beam intensity decays. But the upward first-order phase transition can occur too, since $\mathbf{a}$ is not just a function of beam intensity, but will e.g. also vary with bunch length. Almost instantaneous triggering of the electron cloud can also be expected during filling. Even though this situation has not been modeled here, the mechanism would be similar.

\section{Discussion and conclusions}

The present model has been limited to equal spacing between bunches for simplicity reasons. For larger bunch spacing, the sensitivity of the electron cloud evolution to the described effect should be larger. It is in fact the survival of the electron cloud across surprisingly long gaps $[10,11]$ that stimulated the thought that the presence of positive ions may be aiding this survival.

The new approach introduced in Ref. 8 of modeling the complex multipacting phenomenon with just two parameters has been extended here with one more parameter to take into account the possible role of the desorbed gas in reducing the electron-cloud decay rate. The mechanism considered here and the one described in Ref. 9, both lead to multipacting-enhancement by desorbed gas, and to first order phase transitions. Both types of mechanisms will probably be present to some extent. 
The actual observation of first-order phase transitions in RHIC, together with the simplified cases analyzed here and in Ref. 9, predicting such transitions, lend some credence to the long-held suspicion that the evolved gas is not merely a byproduct of multipacting, but also an active participant in enhancing the electron-cloud evolution. Increased pumping would therefore not only mitigate the effects of outgassing due to multipacting, but it would also diminish the growth of the electron cloud itself.

Further work, including more experiments, improvement of simulation codes, and development of countermeasures will be essential in overcoming present limitations. We hope that the simple approach used here will provide some intuitive guidance for such efforts.

\section{References}

1) W. Fischer and U. Iriso, "Bunch Patterns and Pressure Rise in RHIC”, Proceedings of EPAC'04, pg 914, MOPLT164.

2) http://slap.cern.ch/collective/ecloud02/ and http://icfa-ecloud04.web.cern.ch/icfa-ecloud04/

3) F.Zimmermann, G.Rumolo, M.Furman, L.Wang, M.Blaskiewicz, et al. Review and Comparison of Simulation Codes Modeling Electron-Cloud Build Up and Instabilities, Proc. of EPAC'04, Lucerne, 2004. See also http://wwwslap.cern.ch/collective/ecloud02/ecsim

4) W. Fischer, U. Iriso, and E. Mustafin. "Electron cloud driven vacuum instability" Proc. of ICFA Workshop on High Intensity and High Brightness Hadron Beams, Bensheim, October 2004.

5) O. Grobner, "Vacuum aspects for an LHC upgrade", Proc. of CARE HHH-2004 Workshop, Geneva, November 2004.

6) U. Iriso and S. Peggs, "Electron Cloud phase transitions", C-AD/AP/147, April 2004.

7) Rumolo, F. Zimmermann, Interplay of Ionization and Sputtering with the Electron Cloud, CERN-SL-2001-014-AP.

8) U. Iriso and S. Peggs, Phys. Rev. ST Accel. Beams 8, 024403 (2005)

9) U. Iriso and S. Peggs, to be published.

10) S. Y. Zhang "Secondary Electron Survival in the Bunch Gap", C-AD/AP/48, April 2001

11) G. Arduini, T. Bohl, K. Cornelis, W. Hšfle, E. MŽtral, F. Zimmermann, , Beam Observations With Electron Cloud in the CERN PS \& SPS Complex http://mafurman.lbl.gov/ECLOUD04_proceedings/arduini-ga_ecloud04_v2.pdf, Proceedings ECLOUD'04 